\title{
Study of Temperature Dependent Dialectical Properties of One System Ceramics using BNT-BZ
}

\author{
Aloke Verma \\ Assistant Professor, Department of Physics, Kalinga University, New Raipur, (C.G.), India, Pin: 492101
}

\begin{abstract}
In this paper, Study of temperature dependent dialectical properties of one system ceramics like BNT-BZ. Lead-free BNT-BZ ceramics were successfully prepared using the conventional mixed-oxide method. The samples were sintered for 2 hrs at temperatures lower than $1,000^{\circ} \mathrm{C}$. The density of the BNT-BZ samples was at least $95 \%$ of the theoretical values. The scanning electron microscopy micrographs showed that small grains were embedded between large grains, causing a relatively wide grain size distribution. The density and grain size increased with increasing $\mathrm{Zr}$ concentration. A peak shift in $\mathrm{X}$-ray diffraction patterns as well as the disappearance of several hkl reflections indicated some significant crystal-structure changes in these materials. Preliminary crystal-structure analysis indicated the existence of phase transition from a rhombohedral to an orthorhombic structure. The dielectric and ferroelectric properties were also found to correlate well with the observed phase transition.
\end{abstract}

Keywords: ceramics; X-ray diffraction; dielectric properties; ferroelectricity.

\section{Introduction}

Piezoelectric materials are commonly used in sensor and actuator technologies due to their unique ability to couple electrical and mechanical displacements that is to change electrical polarization in response to an applied mechanical stress or mechanically strain in response to an applied electric field. The most widely used piezoelectric ceramic is lead zirconate titanate [PZT]. Lead-based $\mathrm{PbTiO}_{3}-\mathrm{PbZrO}_{3}$ solid solutions have dominated the market of actuator and sensor materials due to their excellent ferroelectric and piezoelectric properties. In particular, a compositional ratio of $\mathrm{Zr} / \mathrm{Ti}$ of around 52/48 showed the morphotropic phase boundary between a tetragonal and a rhombohedral phase, where enhanced polarizability and optimum domain orientation were observed. However, $\mathrm{PbO}$ loss during hightemperature processes is considered to be environmental pollution with additional problems of recycling and waste disposal and critical disadvantage of PZT is that it contains more than 60 percent lead $(\mathrm{Pb})$ by weight. Therefore, researchers have attempted to develop new lead-free smart materials in order to replace the lead-based ones. BT is one example of the most commonly used lead-free material for capacitors and actuators due to its inherent ferroelectric nature. However, its main disadvantage is the narrow working temperature; therefore, the use of a BT-BZ solid solution with the addition of $\mathrm{Zr}$ up to $30 \%$ mole was investigated. The materials were found to exhibit a composition-induced phase transition from normal to relaxor ferroelectric with a higher dielectric constant than both PZT and BT. This allowed the materials to be used over a broader temperature range. Following these studies, this paper was aimed to study BNT-BZ solid solutions with the addition of a $\mathrm{Zr}$ concentration from $0.20,0.35,0.40,0.45,0.60$, and 0.80 mole fractions. The relationship between the phase, crystal structure, and electrical properties is investigated and discussed.

\section{Experimental Methods}

BNT-BZ compositions were prepared using the mixed-oxide method. The calcined BNT-BZ powders were then ball milled again for $6 \mathrm{~h}$ and uniaxially pressed at a pressure of $5.5 \mathrm{MPa}$ with a few drops of 3 wt.\% polyvinyl alcohol to bind it into disks of $10-\mathrm{mm}$ diameter and 1 - to $1.5-\mathrm{mm}$ thickness. The disks were the sintered at $900^{\circ} \mathrm{C}$ for $2 \mathrm{hrs}$, except for the sample with 0.20 mole fraction $\mathrm{Zr}$ which was sintered at $950^{\circ} \mathrm{C}$ for 2 hrs, in air. The preliminary crystal structure details were calculated using the Powder Cell program, which is based on the X-ray diffraction pattern of lead-free BNT-BZ ceramics. The bulk densities of the sintered ceramics were measured using Archimedes' method. The theoretical density was approximated from the unit cell size and its constituent ions. Scanning electron microscopy [SEM] was used to observe the microstructure of the ceramics. To prepare the SEM samples, they were well-polished and thermally etched for $15 \mathrm{~min}$ at $750^{\circ} \mathrm{C}$. The average grain size was then evaluated from these SEM images. Results and discussion X-ray diffraction patterns of BNT are shown in Figure 1. The NT-BZ phase could be matched with pure BNT for the rhombohedral space group R3c. With the presence of $\mathrm{Zr}$, all reflection peaks systematically shifted to angles lower than $2 \theta$. Accompanying the shift, intensities of some diffraction peaks such as (012) and (202) were reduced, indicating that lattice distortion alongside unit cell expansion has occurred. The refinement of the X-ray diffraction patterns was carried out. 
International Journal of Science and Research (IJSR)

ISSN (Online): 2319-7064

Index Copernicus Value (2015): 78.96 | Impact Factor (2015): 6.391

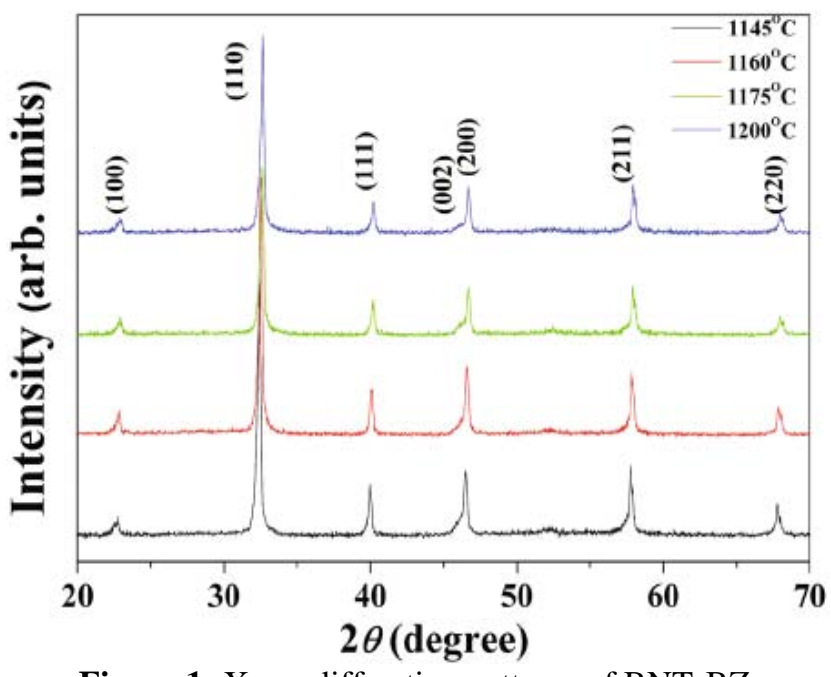

Figure 1: X-ray diffraction patterns of BNT-BZ

BNT-BZ ceramics containing Zr from 0.2 to 0.6 possessed a rhombohedral structure with increased lattice parameters. The increase in the value of the interaxial angle caused the structure to be close to cubic, which resulted in the disappearance of certain reflections. For $\mathrm{Zr}=0.8$, Figures 1 and 2 showed an apparent splitting of the (104) and (300) peaks in the original rhombohedral structure. This finding was rather in partial agreement with the orthorhombic structure previously obtained for BNT-BZ. Hence, for this BNT-BZ solid solution ceramic system, the structure changed from rhombohedral to orthorhombic when the $\mathrm{Zr}$ concentration exceeded 0.6 mole fraction. The exact phasetransition composition is currently being investigated. All BNT-BZ ceramics had experimental density values in the range of 5.8 to $6.1 \mathrm{~g} / \mathrm{cm} 3$ as shown in Table 1 which corresponded to the relative densities of around $95 \%$ of the theoretical densities. For the 0.20 mole fraction of $\mathrm{Zr}$, the sample was sintered at $950^{\circ} \mathrm{C}$ for $2 \mathrm{~h}$ due to the influence of a high $\mathrm{Ti}$ concentration. As the amount of $\mathrm{Zr}$ increased, the sintering temperature could be lowered to $900^{\circ} \mathrm{C}$. This seemed to be a typical behavior of solid solutions whose melting points might be lowered by adding $\mathrm{Zr}$ as a deducted form of the lattice expansion. The difference in sintering behavior could also be observed from the microstructure of BNT-BZ ceramics; all samples were dense with welldefined grains. The ceramic containing $\mathrm{Zr}=0.2$ possessed an average grain size of about $0.8 \mu \mathrm{m}$, whereas the presence of $\mathrm{Zr}$ ions generally caused the grain size to increase. The enhanced ability for ionic diffusion in BNT-BZ ceramics seemed to support the possible lowering of the melting point of these solid solutions. In general, increasing $\mathrm{Zr}$ concentration in BNT-BZ ceramics caused a gradual drop off in dielectric constant with a slight drop off in dielectric loss. This behavior was in agreement with other systems with isovalent additives.

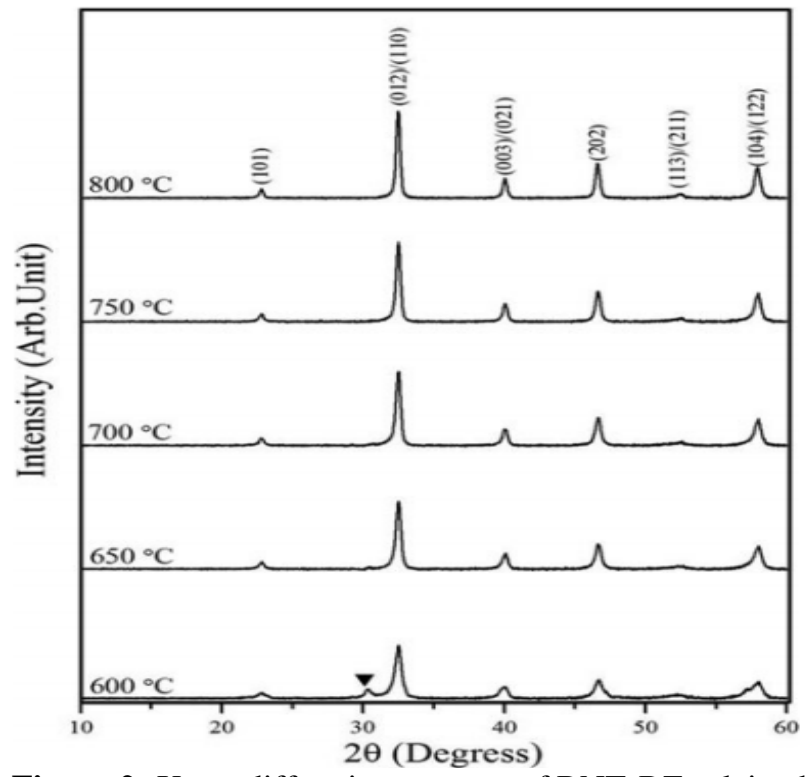

Figure 2: X-ray diffraction patterns of BNT-BZ calcined powders at various temperatures.

In addition, the replacement of larger $\mathrm{Zr}$ ions may also cause the dipoles to be poorly induced due to limited ionic movement. This decreasing trend was observed through the sample with a composition of $\mathrm{Zr}=0.8$, whose structure was orthorhombic. It seemed that the effect of ionic size and limited ionic movement in the perovskite structure of this compound had a greater influence on the dielectric properties than the change in the crystal structure in their unit-all dimensions. Figure 4 illustrates the breakdown field strengths of BNT-BZ ceramics, respectively. The hysteresis loops were obtained at the maximum applied electric field of $20 \mathrm{kV} / \mathrm{cm}$ and a frequency of $50 \mathrm{~Hz}$. Limited domain reorientation might also be the cause of poor hysteresis loops for these compositions. For samples with $\mathrm{Zr}=0.6$ and 0.8 , the loops showed higher values of remnant polarization though they were still unsaturated. 


\section{International Journal of Science and Research (IJSR) ISSN (Online): 2319-7064}

Index Copernicus Value (2015): 78.96 | Impact Factor (2015): 6.391

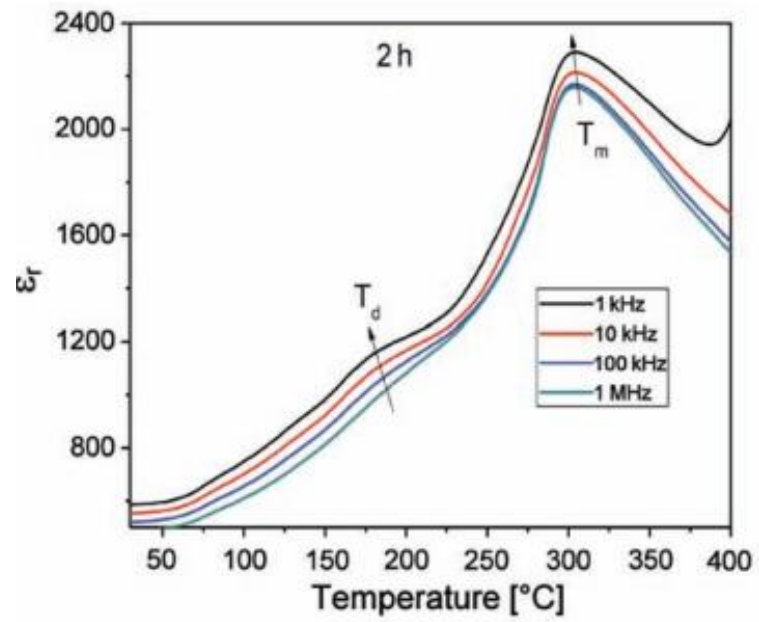

a)

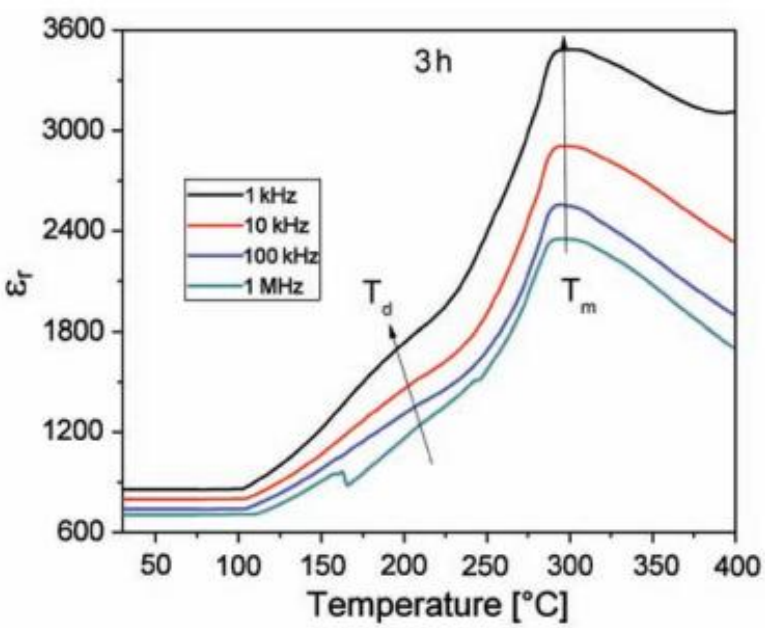

b)

Figure 3: Temperature and frequency dependent dielectric study of BNT ceramics sintered at $1100{ }^{\circ} \mathrm{C}$ for: a) 2 and b) 3 hours

This seemed to show the approximate transition point between the rhombohedral and orthorhombic structures. This was supported by an increase in the breakdown field strength for the $\mathrm{Zr}=0.8$ composition, which was partly due to the effect of a different crystal structure in this series of materials. Hence, this study showed that the observed dielectric and ferroelectric properties of BNT-BZ ceramics largely depended on compositional and crystal structure changes. Optimization of these properties could be achieved by fine-tuning the composition for specific applications.

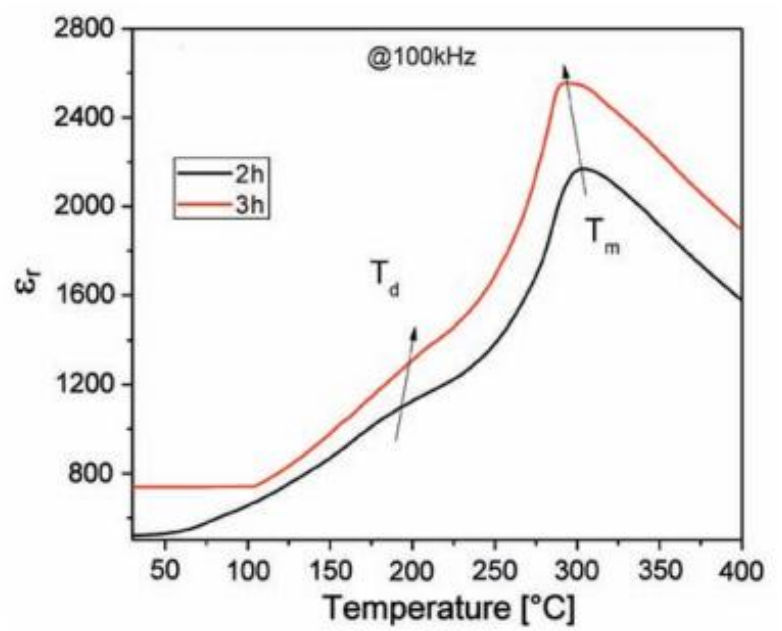

Figure 4: Variation of dielectric constant with sintering temperature and time

\section{Conclusions}

This study showed that electrical properties of BNT-BZ ceramics could be further improved by fine-tuning their composition for certain applications. The BNT ceramic pellets were sintered at $1100{ }^{\circ} \mathrm{C}$ for two different times. The scanning electron micrographs of the sintered pellet showed well developed rectangular grains and the increased density of the pellet with the increase in the sintering time. The temperature dependent dielectric study showed that two types of phase transitions occurred in both samples. The dielectric analysis also showed that the transition temperature increases with frequency, thus confirming a diffuse phase transition behavior.

\section{References}

[1] Cross LE, Newnham RE: History of ferroelectrics. J Am Ceram 1987, 11:289-305.

[2] G.A. Smolenskii, A. Agranovskaya I, N.N. Krainic, "New ferroelectrics of complex composition IV", Sov. Phys.-Solid State, 2 (1961) 2651-2654.

[3] H. Nagata, T. Takenakan, "Lead-free piezoelectric ceramics of (Bi1/2Na1/2)TiO3-BiFeO3 system", Key Eng. Mater., 169-170 (1999) 37-40.

[4] Haertling GH: Ferroelectric ceramics: history and technology. J Am Ceram 1999, 82:797.

[5] J. Suchanicz, K. Roleder, A. Kania, J. Handerek, "Electrostrictive strain and pyroeffect in the region of phase coexistence in Na0.5Bi0.5TiO3, Ferroelectrics, 77 (1988) 107-110.

[6] J. Yeongho, Y. Juhyun, L. Sangho, "Piezoelectric characteristics of low temperature sintering $\mathrm{Pb}(\mathrm{Mn} 1 / 3 \mathrm{Nb} 2 / 3) \mathrm{O} 3-\mathrm{Pb}(\mathrm{Ni1} / 3 \mathrm{Nb} 2 / 3) \mathrm{O} 3-$

$\mathrm{Pb}(\mathrm{Zr} 0.50 \mathrm{Ti} 0.50) \mathrm{O} 3$ according tothe addition of $\mathrm{CuO}$ and Fe 2O3", Sens. Actuators A: Phys., 135 (2007) 215-219.

[7] Jaffe B, Cook WR, Jaffe H: Piezoelectric Ceramics London: Academic Press; 1971.

[8] Jones GO, Thomas PA: Investigation of the structure and phase transition in the novel A-site substituted distorted perovskite compound $\mathrm{Na} 0.5 \mathrm{Bi} 0.5 \mathrm{TiO} 3$. Acta Cryst 2002, B58:168-178.

[9] Jones GO, Thomas PA: The tetragonal phase of $\mathrm{Na} 0.5 \mathrm{Bi} 0.5 \mathrm{TiO} 3$ - a new variant of the perovskite structure. Acta Crysta 2000, B58:426-430.

[10] K. Roleder, J. Suchanicz, A. Kania, “Time-dependence of electric permittivity in $\mathrm{Na} 0.5 \mathrm{Bi} 0.5 \mathrm{TiO} 3$ singlecrystals", Ferroelectrics, 89 (1989) 1-5.

[11] Kraus W, Nolze G: POWDER CELL- a program for the representation and manipulation of crystal structures and calculation of the resulting X-ray powder patterns. J Appl Cryst 1996, 29:301-303.

[12] Lily Kumari K, Prasad K, Yadav KL: Dielectric and impedance study of leadfree ceramic: (Na0.5Bi0.5)ZrO3. J Mater Sci 2007, 42:6252-6259.

[13] Moulson AJ, Herbert JM: Electroceramics: Materials, Properties, Applications Chichester: Wiley; 2003. 
[14] P. Marchet, E. Boucher, V. Dorcet, "Dielectric properties of some lead free $\mathrm{Na}$ 0.5Bi0.5TiO3-BiScO3 and $\mathrm{Na}$ 0.5Bi0.5TiO3-BiFeO3 ceramics", J. Eur. Ceram. Soc., 26 (2006) 3037-3041.

[15] Panda PK: Review: environmental friendly lead-free piezoelectric materials. J Mater Sci 2009, 44:50495062.

[16] Q. Xu, X.L. Chen, W. Chen, S.T. Chen , B. Kim, J. Lee, "Synthesis, ferroelectric and piezoelectric properties of some $(\mathrm{Na} 0.5 \mathrm{Bi} 0.5) \mathrm{TiO} 3$ system compositions", Mater. Lett., 59 (2005) 2437-2441.

[17] Rachakom et al., Crystal structure and electrical properties of bismuth sodium titanate zirconate ceramics, Nanoscale Research Letters 2012,

[18] S. Senda, M. Jean-Pierre, "Relaxorbehavior of low lead and lead free ferroelectric ceramic of the (Na0.5Bi0.5)TiO3-PbTiO3 and (Na0.5Bi0.5)TiO3(K0.5Bi0.5)TiO3 systems", J. Eur. Ceram. Soc., 21 (2001) 1333-1336.

[19] Shannon RD: Revised effective ionic radii and systematic studies of interatomic distances in halides and chalcogenides. Acta Cryst 1976, A32:751-767.

[20] Shrout TR, Zhang SJ: Lead-free piezoelectric ceramics: alternatives for PZT? J Electroceram 2007, 19:111-124.

[21] T. Takenaka, K. Maruyama, K. Sakata, "(Na0.5Bi0.5)TiO3-BaTiO3 system for lead free piezoelectric ceramics”, Jpn. J. Appl. Phys., 30 (1991) 2236-2239.

[22] T. Takenaka, K. Sakata, "Dielectric, piezoelectric and pyroelectric properties of (BiNa)1/2TiO3-based ceramics", Ferroelectrics, 95 (1989) 153-156.

[23] T. Takenaka, K. Sakata, K. Toda, "Piezoelectric properties of (Bi1/2Na1/2)TiO3-based ceramics", Ferroelectrics, 106 (1990) 375-380.

[24] Takenaka T, Nagata H, Hiruma Y, Yoshii Y, Matumono K: Lead-free piezoelectric ceramics based on perovskite structures. 2007, 19:259-265.

[25] Tang XG, Chew K-H, Chan HLW: Diffuse phase transition and dielectric tenability of Ba(Zry, Ti1-y)O3 relaxor ferroelectric ceramics. Acta Materialia 2004, 52:5177-5183.

[26] Watcharapasorn A, Jiansirisomboon S, Tunkarisi T: Effects of dysprosium oxide addition in bismuth sodium titanate ceramics. J Electroceram 2008, 21:613-616.

[27] Watcharapasorn A, Jiansirisomboon S: Dielectric and piezoelectric properties of zirconium-doped bismuth sodium titanate ceramics. Adv Mater Res 2008, 55:133-136.

[28] Y. Zupei, C. Xiaolian, Z. Rui, "Fabrication and electrical characterization of PMN-PZN-PZT ceramic transformers", Mater. Sci. Eng. B, 138 (2007) 277283.

[29] Yu Z, Ang C, Guo R, Bhalla AS: Dielectric properties of Ba(Ti1-xZrx)O3. Mater Lett 2007, 61:326-329.

[30] Yu Z, Ang C, Guo R, Bhalla AS: Piezoelectric and strain properties of Ba (Ti1-x, Zrx)O3 ceramics. J Appl Phys 2002, 92:1489-1493. 